LINEAR AND NON-LINEAR THEORY OF

GENERALIZED FUNCTIONS AND ITS APPLICATIONS
BANACH CENTER PUBLICATIONS, VOLUME 88
INSTITUTE OF MATHEMATICS
POLISH ACADEMY OF SCIENCES
WARSZAWA 2010

\title{
SHEAF THEORY AND REGULARITY. APPLICATION TO LOCAL AND MICROLOCAL ANALYSIS
}

\author{
JEAN-ANDRÉ MARTI \\ Equipe Analyse Algébrique Non Linéaire \\ Laboratoire CEREGMIA \\ Université des Antilles et de la Guyane \\ 97275 Schoelcher Cedex, Martinique (France) \\ E-mail: jean-andre.marti@univ-ag.fr
}

\begin{abstract}
A review of some methods in sheaf theory is presented to make precise a general concept of regularity in algebras or spaces of generalized functions. This leads to the local analysis of the sections of sheaves or presheaves under consideration and then to microlocal analysis and microlocal asymptotic analysis.
\end{abstract}

1. Introduction. We are going to present in this article a review of certain methods in sheaf theory and to discuss the notion of regularity in algebras or spaces of generalized functions. We dissociate the microlocal analysis of the sections of sheaves or presheaves under consideration into so-called frequential microlocal analysis and a microlocal asymptotic analysis.

The frequential microlocal analysis based on the Fourier transform leads to the study of propagation of singularities under only linear operators (including pseudodifferential operators) in the theories described further on. However the study has been extended to certain non-linear cases in the classical theories involving Sobolev's techniques. The microlocal asymptotic analysis is a new spectral study of singularities which gives some results involving nonlinear operations.

In terms of sheaf theory, the notion of regularity in algebras or spaces of generalized functions can be formulated in a general way. If $\mathcal{A}$ is a presheaf of algebras or vector spaces on a topological space $X, \mathcal{B}$ is a subpresheaf of $\mathcal{A}$ and $\Omega$ is an arbitrary open set in $X$, then $\mathcal{B}(\Omega)$ can be considered as the space or algebra of some regular elements of

2000 Mathematics Subject Classification: Primary 35A27; Secondary 35A18, 46F30, 35D10.

Key words and phrases: sheaf theory, algebras of generalized functions, localization, microlocalization and wave front sets, nonlinear partial differential equations.

The paper is in final form and no version of it will be published elsewhere. 
$\mathcal{A}(\Omega)$. This leads to the notion of $\mathcal{B}$-singular support refining the notion of support of a section $u \in \mathcal{A}(\Omega)$, under the following localization principle:

$\left(F_{1}\right)$ if $u$ and $v$ are global sections of $\mathcal{A}$ which agree on every open set of a family $\left(\Omega_{i}\right)_{i \in I}$ of open sets in $X$, then they agree on the union $\bigcup_{i \in I} \Omega_{i}$.

One can give many examples of such situations in the framework of theories of generalized functions, e.g. in the theory of distributions [38 or in the theories of Colombeau-type algebras [1, 7, 8, 9, 10. Differential or pseudo-differential operators are special cases of so called $\mathcal{B}$-compatible operators giving estimates on the behaviour of $\mathcal{B}$-singular supports.

2. Microlocal and spectral asymptotic analysis. We are going to restrict our study to the following two subjects: the frequential microlocal analysis and the spectral asymptotic analysis.

2.1. The frequential microlocal analysis. In this subject we are interested in considering the following three cases:

- Distribution spaces and Sobolev-type regularity. The wave front set $W F(u)$ of $u \in \mathcal{D}^{\prime}(\Omega)$ is a well known refinement of its singular support [21. This leads to the study of propagation of singularities of distributions under differential or pseudo-differential actions. Such studies even exist for nonlinear operators in a classical framework involving some spaces of Sobolev type. In Section 8 of [23, Hörmander uses the results on microlocal $H_{(s)}^{\text {loc }}$-regularity of nonlinear operations for tempered distributions in $\mathcal{S}^{\prime}\left(\mathbb{R}^{n}\right)$ to discuss semi-linear equations, following Rauch 35. By means of paradifferential techniques some general results for quasilinear equations are given in Bony [4. Then some fully nonlinear equations are discussed from a general result on propagation of singularities for pseudodifferential operators and a Bony's linearization theorem. Precise propagation results for hyperbolic second order semi-linear equations are obtained. Extensions of the previous results can be found in works of Beals [2, 3] and Bony [5].

- $\mathcal{G}^{r}$-Colombeau-type algebra and $\mathcal{G}^{r, \mathcal{R}}$ or $\mathcal{G}^{r, \mathcal{R}, L}$-regularity. To give a microlocal study of generalized functions Nedeljkov, Pilipović and Scarpalézos [31, 37] take $\mathcal{A}=\mathcal{G}$, the Colombeau simplified algebras and $\mathcal{B}=\mathcal{G}^{\infty}$ as the "first" regular subsheaf of $\mathcal{G}$ defined by Oberguggenberger 32 . The crucial point was the conservation of the power of Lemma 8.1.1 in 21] leading to the definition of the generalized wave front set of $u \in \mathcal{G}(\Omega)$ denoted $W F_{g}(u)$. Recently A. Delcroix has extended in [12 the $\mathcal{G}^{\infty}$ regularity to a so called $\mathcal{G}^{\mathcal{R}}$ regularity, which still preserves the statements of the above quoted lemma, and gives a $\mathcal{G}^{\mathcal{R}}$-frequential microanalysis. The Fourier transform is still the main tool involved in other generalized cases, where the $\mathcal{G}^{\infty}$-regularity is subjected to an additional condition (such as an estimate on the growth of derivatives) characterizing a special property such as to belong to an analytic, Gevrey or $\mathrm{C}^{L}$ class in Hörmander sense ([21], Section 8.4). Such cases are studied by Pilipović, Scarpalézos and Valmorin [34, Marti [29], Bouzar and Benmeriem [6]. They are special cases of the more general one obtained when taking $\mathcal{A}=\mathcal{G}^{r}$ which extends the Colombeau algebra $\mathcal{G}$ and $\mathcal{B}=\mathcal{G}^{r, \mathcal{R}, L}$ which generalizes all the previous regularity cases. 
- Functional space $\mathcal{L}\left(\mathcal{G}_{c}(\cdot), \widetilde{\mathbb{C}}\right)$ and $\mathcal{G}$-regularity. Many results on propagation of singularities and pseudo-differential techniques have been obtained during the last years by various authors, inspired by the classical theories (see e.g. [15, 18, 19, 24, 25, 26]). For instance, if $u \in \mathcal{G}(\Omega)$, Hörmann and Garetto [18 have obtained characterizations of $W F_{g}(u)$ in terms of intersections of certain domains corresponding to pseudo-differential operators analogously to Hörmander's characterizations of $W F(u)$ for $u \in \mathcal{D}^{\prime}(\Omega)[22$. However these deep results are limited mainly to linear cases, at least in the framework described above. Even in case $\mathcal{A}$ is a sheaf of factor algebras, no study is made on the microlocal behaviour of singularities under nonlinear operations by means of frequential methods based on the Fourier transform.

2.2. The spectral asymptotic analysis. We are going to give the meaning of an asymptotic analysis [14, 27, 28] when $\mathcal{A}(\Omega)=\mathcal{G}(\Omega)$. Let $\mathcal{F}$ be a subsheaf of vector spaces (or algebras) of $\mathcal{G}$. First we define the sheaf $\mathcal{B}$ such that $\mathcal{B}(V)$, for every open set $V$ in $\mathbb{R}^{n}$, is the space of all $u=\left[u_{\varepsilon}\right] \in \mathcal{A}(V)$ such that $u_{\varepsilon}$ has a limit in $\mathcal{F}(V)$ as $\varepsilon$ tends to 0 . Then we define $\mathcal{O}_{\mathcal{G}}^{\mathcal{F}}(u)$ as the set of all $x \in \Omega$ such that $u$ agrees with a section of $\mathcal{B}$ above some neighbourhood of $x$. In turn, the $\mathcal{F}$-singular (or $\mathcal{B}$-singular) support of $u$ is defined by $\mathcal{S}_{\mathcal{G}}^{\mathcal{F}}(u)=\Omega \backslash \mathcal{O}_{\mathcal{G}}^{\mathcal{F}}(u)$ (or $\mathcal{S}_{\mathcal{G}}^{\mathcal{B}}(u)=\Omega \backslash \mathcal{O}_{\mathcal{G}}^{\mathcal{B}}(u)$ ). For fixed $x$ and $u$, we denote by $N_{x}(u)$ the set of all $r \in \mathbb{R}_{+}$such that $\varepsilon^{r} u_{\varepsilon}$ tends to a section of $\mathcal{F}$ above some neighbourhood of $x$. The $\mathcal{F}$-singular spectrum of $u$ is defined as the set of all $(x, r) \in \Omega \times \mathbb{R}_{+}$such that $r \in \mathbb{R}_{+} \backslash N_{x}(u)$. This leads to a spectral decomposition of the $\mathcal{F}$-singular support of $u$.

This asymptotic analysis is extended to $(\mathcal{C}, \mathcal{E}, \mathcal{P})$ algebras. This gives the general asymptotic framework, in which the net $\left(\varepsilon^{r}\right)_{\varepsilon}$ is replaced by any net $a$ satisfying some technical conditions, leading to the concept of the $(a, \mathcal{F})$-singular parametric spectrum. The main advantage is that this asymptotic analysis is compatible with the algebraic structure of the presheaf $\mathcal{F}$ asymptotically associated to $(\mathcal{C}, \mathcal{E}, \mathcal{P})$ algebras. Thus the $(a, \mathcal{F})$-singular asymptotic spectrum inherits good properties with respect to nonlinear operations when $\mathcal{F}$ is a presheaf of topological algebras. Moreover, even when $\mathcal{F}$ is a presheaf (or sheaf) of vector spaces (for instance $\mathcal{F}=\mathcal{D}^{\prime}$ ), some results on microlocal analysis are still obtained for nonlinear operations (see paragraph 4.3.1) on $\left(a, \mathcal{D}^{\prime}\right)$-singular asymptotic spectrum of powers of $\delta$ functions. In [14], various examples of propagation of singularities through nonlinear differential operators are given, connected to some results of Oberguggenberger, Rauch, Reeds and Travers [33, 36, 39].

\section{Sheaf interpretation of regularity and local analysis of generalized func-}

tions. Let us consider the following sequence of sheaf embeddings, defined for each $\Omega$ open set in $\mathbb{R}^{n}$ by

$$
\mathrm{C}^{\infty}(\Omega) \rightarrow \mathcal{D}^{\prime}(\Omega) \rightarrow \mathcal{G}(\Omega)
$$

where $\mathcal{G}(\Omega)$ is the Colombeau algebra. Each term of the sequence can be considered as the $\mathcal{B}(\Omega)$ regular space or algebra of the following algebra or space $\mathcal{A}(\Omega)$. It is the basis for a local analysis of the elements in $\mathcal{A}(\Omega)$. The purpose of this section is to localize the singularities of some generalized functions and to study their behaviour under a general $\mathcal{B}$-compatible operator. We refer the reader to [20] for more details on the sheaf theory involved in the sequel. 
3.1. The basic definitions. We recall that

$1^{\circ} \mathcal{A}$ is a given sheaf of sets (or presheaf satisfying, in addition, the localization principle $\left(F_{1}\right)$ ) over a topological space $X$.

$2^{\circ}$ is a given subsheaf (or subpresheaf) of $\mathcal{A}$.

Definition 3.1. For an arbitrary open set $\Omega$ in $X$, elements of $\mathcal{B}(\Omega)$ are considered as regular and are called $\mathcal{B}$-regular elements of $\mathcal{A}(\Omega)$.

Definition 3.2. For an arbitrary open set $\Omega$ in $X$, an element $u \in \mathcal{A}(\Omega)$ is called $\mathcal{B}$-regular at $x \in \Omega$ if there is an open neighbourhood $V$ of $x$ such that the restriction $\left.u\right|_{V}$ of $u$ to $V$ belongs to $\mathcal{B}(V)$.

Definition 3.3. By $\mathcal{O}_{\mathcal{A}}^{\mathcal{B}}(u)$, denote the set of all $x \in \Omega$ such that $u$ is $\mathcal{B}$-regular at $x$, i.e.

$$
\mathcal{O}_{\mathcal{A}}^{\mathcal{B}}(u)=\left\{x \in \Omega: \exists V \in \mathcal{V}_{x},\left.u\right|_{V} \in \mathcal{B}(V)\right\},
$$

where $\mathcal{V}_{x}$ denotes the family of all open neighbourhoods of $x$.

Definition 3.4. For an arbitrary open set $\Omega$ in $X$ and a section $u \in \mathcal{A}(\Omega)$, we define the $\mathcal{B}$-singular support $\mathcal{S}_{\mathcal{A}}^{\mathcal{B}}(u)$ of $u$ by

REMARK 3.5.

$$
\mathcal{S}_{\mathcal{A}}^{\mathcal{B}}(u)=\Omega \backslash \mathcal{O}_{\mathcal{A}}^{\mathcal{B}}(u) .
$$

a) We recall that the presheaf $\mathcal{A}$ becomes a sheaf when adding the gluing principle $\left(F_{2}\right)$ to the localization one $\left(F_{1}\right)$ given in Introduction.

$\left(F_{2}\right)$ Let $\left(\Omega_{i}\right)_{i \in I}$ be a family of open sets in $X$ and $\left(v_{i}\right)_{i \in I}$ a family of sections $v_{i} \in \mathcal{A}\left(\Omega_{i}\right)$ such that, for each $(i, j) \in I \times I,\left.v_{i}\right|_{\Omega_{i} \cap \Omega_{j}}=\left.v_{j}\right|_{\Omega_{i} \cap \Omega_{j}}$.

Then there exists $v \in \mathcal{A}\left(\bigcup_{i \in I} \Omega_{i}\right)$ such that, for each $i \in I,\left.v\right|_{\Omega_{i}}=v_{i}$.

However the $\left(F_{2}\right)$ principle is not needed to define the $\mathcal{B}$-singular support of a section $u \in \mathcal{A}(\Omega)$ for an arbitrary open set $\Omega$ in $X$.

b) the set $\mathcal{S}_{\mathcal{A}}^{\mathcal{B}}(u)$ is always a closed subset of $\mathcal{S}_{\mathcal{A}}^{\{0\}}(u)=\operatorname{supp}(u)$.

c) In general, the set $\mathcal{O}_{\mathcal{A}}^{\mathcal{B}}(u)$ is not the largest open set $\mathcal{O}$ such that $\left.u\right|_{\mathcal{O}}$ is in $\mathcal{B}(\mathcal{O})$ except in the following two cases:

$1^{\circ}$ if $\mathcal{B}=\{0\}\left(\right.$ then $\left.\mathcal{S}_{\mathcal{A}}^{\mathcal{B}}(u)=\operatorname{supp}(u)\right)$;

$2^{\circ}$ if $\mathcal{B}$ is a sheaf (even if $\mathcal{A}$ is only a prehesaf).

The elementary algebraic properties of the $\mathcal{B}$-singular support are given by the following (see [30]):

Proposition 3.6. Suppose that $\mathcal{B}$ and $\mathcal{A}$ are $(a)$ presheaves of $\mathbb{K}$-vector spaces or $(b)$ algebras. Let $\left(u_{j}\right)_{1 \leq j \leq p}$ be an arbitrary finite family of elements in $\mathcal{A}(\Omega)$ and $\left(\lambda_{j}\right)_{1 \leq j \leq p}$ be an arbitrary finite family of elements in $\mathbb{K}$. Then

$$
\mathcal{S}_{\mathcal{A}}^{\mathcal{B}}\left(\sum_{1 \leq j \leq p} \lambda_{j} u_{j}\right) \subset \bigcup_{1 \leq j \leq p} \mathcal{S}_{\mathcal{A}}^{B}\left(u_{j}\right)
$$

in cases $(a)$ and $(b)$ and, in addition in case $(b)$,

$$
\mathcal{S}_{\mathcal{A}}^{\mathcal{B}}\left(\prod_{1 \leq j \leq p} u_{j}\right) \subset \bigcup_{1 \leq j \leq p} \mathcal{S}_{\mathcal{A}}^{B}\left(u_{j}\right) .
$$

In particular, in case $(b)$, we have $\mathcal{S}_{\mathcal{A}}^{\mathcal{B}}\left(u^{p}\right) \subset \mathcal{S}_{\mathcal{A}}^{B}(u)$. 
3.2. Localization of singularities. Let $\Omega$ be a given open subset of $X$. A presheaf operator $A$ in $\mathcal{A}(\Omega)$ is defined as a presheaf morphism $\mathcal{A}(\Omega) \rightarrow \mathcal{A}(\Omega)$ compatible with restrictions. More precisely if $\mathcal{O}_{\Omega}$ denotes the category of all open sets in $\Omega, A$ may be given by a collection $\left(A_{V}\right)_{V \in \mathcal{O}_{\Omega}}$ of mappings $A_{V}: \mathcal{A}(V) \rightarrow \mathcal{A}(V)$ such that for each $V \in \mathcal{O}_{\Omega}$ and $u \in \mathcal{A}(\Omega)$ we have: $\left.A_{\Omega}(u)\right|_{V}=A_{V}\left(\left.u\right|_{V}\right)$. Thus we can simplify the notations and write $A$ instead $A_{V}$ when acting on sections over $V$.

Definition 3.7. Let $A$ be a presheaf operator in $\mathcal{A}(\Omega)$. Then $A$ is said to be locally $\mathcal{B}$-compatible if for every $(x, V, v) \in \Omega \times \mathcal{V}_{x} \times \mathcal{B}(V)$ there is a $W \in \mathcal{V}_{x}$ such that $W \subset V$ and $\left.A(v)\right|_{W} \in \mathcal{B}(W)$.

The following result is proved in [30]:

Proposition 3.8. Suppose the assumptions from Subsection 3.1 are fulfilled. Let $A$ be a presheaf operator in $\mathcal{A}(\Omega)$ locally $\mathcal{B}$-compatible. Then $\mathcal{S}_{\mathcal{A}}^{\mathcal{B}}(A(u)) \subset \mathcal{S}_{\mathcal{A}}^{B}(u)$.

The following weaker form of local $\mathcal{B}$-compatibility are more practical for applications:

Definition 3.9. A presheaf operator $A$ in $\mathcal{A}(\Omega)$ is said to be $\mathcal{B}$-compatible if it maps $\mathcal{B}(V)$ into itself for every open set $V$ of $\Omega$.

Evidently, a $\mathcal{B}$-compatible operator is locally $\mathcal{B}$-compatible. The following simple result is useful:

Proposition 3.10. If a presheaf operator $A$ in $\mathcal{A}(\Omega)$ is $\mathcal{B}$-compatible, then the composition product $A^{p}=\overbrace{A \circ \ldots \circ A}^{p}$ is $\mathcal{B}$-compatible for every $p \in N$.

The following result is proved in [30]:

Proposition 3.11. Suppose that $\mathcal{B}$ is a presheaf of algebras and $\mathcal{A}$ is a presheaf of vector spaces and a $\mathcal{B}$-module. Let, for an arbitrary set of indices $\mathfrak{A},\left(b_{\alpha}\right)_{\alpha \in \mathfrak{A}}$ be a family of elements in $\mathcal{B}(\Omega),\left(A_{\alpha}\right)_{\alpha \in \mathfrak{A}}$ be a family of $\mathcal{B}$-compatible operators in $\mathcal{A}(\Omega)$ and $\left(p_{\alpha}\right)_{\alpha \in \mathfrak{A}}$ be a family of positive integers. Then

(i) for every finite $\mathfrak{A}_{0} \subset \mathfrak{A}$, the operator $\sum_{\alpha \in \mathfrak{A}_{0}} b_{\alpha} A_{\alpha}^{p_{\alpha}}$ is $\mathcal{B}$-compatible in $\mathcal{A}(\Omega)$;

(ii) if $\mathcal{A}$ is a presheaf of algebras, then the mapping $u \mapsto \sum_{\alpha \in \mathfrak{A}_{0}} b_{\alpha}\left(A_{\alpha}(u)\right)^{p_{\alpha}}$ is a $\mathcal{B}$-compatible operator in $\mathcal{A}(\Omega)$.

3.3. Examples: a review of principal cases. We divide this section into two parts.

a) In the first part we give a list of examples of embeddings $\mathcal{B} \rightarrow \mathcal{A}$ :

$$
\begin{array}{llll}
\mathrm{C}^{\infty} \rightarrow \mathcal{D}^{\prime} ; & \mathrm{C}^{L} \rightarrow \mathcal{D}^{\prime} ; & \mathcal{G}^{L} \rightarrow \mathcal{G} ; & \mathcal{G}^{\infty} \rightarrow \mathcal{G} ; \\
\mathcal{G}^{\mathcal{R}} \rightarrow \mathcal{G} ; & \mathcal{G}^{\sigma, \infty} \rightarrow \mathcal{G}^{\sigma} ; & \mathcal{G}^{r, \mathcal{R}} \rightarrow \mathcal{G}^{r} ; & \mathcal{G}^{r, \mathcal{R}, L} \rightarrow \mathcal{G}^{r} .
\end{array}
$$

We recall that here

- $\mathcal{G}$ is the sheaf of Colombeau simplified algebra (see [7]).

- $\mathcal{G}^{\infty}$ is the "regular" subsheaf of $\mathcal{G}$, the sheaf of Colombeau's generalized functions (see [32]). 
- $\mathcal{G}^{\mathcal{R}}$ : in $\left[12\right.$ the $\mathcal{G}^{\infty}$-regularity is extended to a $\mathcal{G}^{\mathcal{R}}$ one. Under certain stability conditions on the set $\mathcal{R}$ given in [12, $\mathcal{G}^{\mathcal{R}}$ is a subsheaf of differential algebras of $\mathcal{G}$ and when $\mathcal{R}$ consists of the set of all bounded sequences, then $\mathcal{G}^{\mathcal{R}}=\mathcal{G}^{\infty}$.

- According to Hörmander's definition given in Subsection 8.4 of [21], $\mathrm{C}^{L}$ denotes the sheaf of $\mathbb{K}$-algebras of $\mathrm{C}^{L}$-class on $\mathbb{R}^{n}(\mathbb{K}=\mathbb{R}$ or $\mathbb{C}$.) associated to an increasing sequence $L_{k}$ of positive numbers such that $L_{0}=1$ and $k \leq L_{k}, L_{k+1} \leq C L_{k}$ for some constant $C$. if $L_{k}=k+1, \mathrm{C}^{L}$ is the sheaf A of analytical functions. If $L_{k}=(k+1)^{a}, a>1, \mathrm{C}^{L}$ is the sheaf $\mathrm{G}_{a}$ of the Gevrey class of order $a$.

- $\mathcal{G}^{L}$ : in [29] one constructs $\mathcal{B}=\mathcal{G}^{L}$ as a special regular sub(pre)sheaf of $\mathcal{A}=\mathcal{G}$, extending in a generalized sense the $\mathrm{C}^{L}$ classes of Hörmander. When taking $L_{k}=k+1$, we obtain the analytic case $\mathcal{G}^{A}$ studied in [34] involving special properties of holomorphic generalized functions which give $\mathcal{G}^{A}$ the sheaf property.

- $\mathcal{G}^{\sigma, \infty}$-local analysis in $\mathcal{G}^{\sigma} \neq \mathcal{G}$ defined in [6] is the sheaf of algebra $\mathcal{G}^{\sigma} \neq \mathcal{G}$ of Gevrey ultradistributions with another asymptotic scale than the Colombeau one. $\mathcal{G}^{\sigma, \infty}$ is the sheaf of algebras of Gevrey ultradistributions.

In order to construct a general model containing all the previous examples we have to combine $\mathcal{R}$ and $L=\left(L_{k}\right)_{k}$ with another parameter: an asymptotic scale $r=\left(r_{\lambda}\right)_{\lambda} \in$ $\left(\mathbb{R}_{+}^{*}\right)^{\Lambda}$. This leads to the following (see [30]):

- $\left\{\begin{array}{l}\mathcal{G}^{r} \text { : sheaf of algebras containing } \mathcal{G} \text { and } \mathcal{G}^{\sigma}, \\ \mathcal{G}^{r, \mathcal{R}} \text { : subsheaf of } \mathcal{R} \text {-regularity in } \mathcal{G}^{r}, \\ \mathcal{G}^{r, \mathcal{R}, L} \text { : subpresheaf of }(\mathcal{R} \text { - } L) \text {-regularity in } \mathcal{G}^{r} .\end{array}\right.$

Taking $\lambda=\varepsilon \in] 0,1], r_{\varepsilon}=\varepsilon$ and $R=R_{+}^{\mathbb{N}}$, we recover the sheaf $G$ of Colombeau simplified algebras.

Taking $\lambda=\varepsilon \in] 0,1], r_{\varepsilon}=\varepsilon$ and $R=B o$ (the set of bounded sequences), we obtain the sheaf of $G^{\infty}$-generalized functions [12].

Taking $\lambda=\varepsilon \in] 0,1], r_{\varepsilon}=e^{\varepsilon^{-\frac{1}{2 \sigma-1}}}$ and $R=R_{+}^{\mathbb{N}}$, we obtain the sheaf of so called $G^{\sigma}$-generalized functions in [6].

Taking $\lambda=\varepsilon \in] 0,1], r_{\varepsilon}=\varepsilon, R=B o$ (the set of bounded sequences), and some $L=\left(L_{k}\right)_{k}$, we obtain the presheaf of $G^{L}$-generalized functions (subpresheaf of $G$ ) [29.

Taking $\lambda=\varepsilon \in] 0,1], r_{\varepsilon}=\varepsilon, R=B o$ and $L_{k}=k+1$, we obtain the sheaf of $G^{A}$-generalized functions (subsheaf of $G$ ) 34 .

Taking $\lambda=\varepsilon \in] 0,1], r_{\varepsilon}=e^{\varepsilon^{-\frac{1}{2 \sigma-1}}}, R=B o$ and $L_{k}=(k+1)^{\sigma}$, we obtain the presheaf of $G^{\sigma, \infty}$-generalized functions (subsheaf of $G^{\sigma}$ ) [6].

b) In the second part we consider two other embeddings:

$$
\mathcal{G} \rightarrow \mathcal{L}\left(\mathcal{G}_{c}, \widetilde{\mathbb{C}}\right) ; \quad \mathcal{F}_{\mathcal{A}_{(\mathcal{C}, \mathcal{E}, \mathcal{P})}} \rightarrow \mathcal{A}_{(\mathcal{C}, \mathcal{E}, \mathcal{P})}
$$

- $\mathcal{G}_{c}^{\prime}=\mathcal{L}\left(\mathcal{G}_{c}, \widetilde{\mathbb{C}}\right): \Omega \rightarrow \mathcal{L}\left(\mathcal{G}_{c}(\Omega), \widetilde{\mathbb{C}}\right)$ is a sheaf of vector spaces: the topological dual of $\mathcal{G}_{c}$ [16. The duality in the Colombeau context is defined when starting from the so-called sharp topology of $\mathcal{G}(\Omega)$ 31]. In [17, $\mathcal{L}\left(\mathcal{G}_{c}(\Omega), \widetilde{\mathbb{C}}\right)$ is endowed with the topology of uniform convergence on bounded subsets of $\mathcal{G}(\Omega)$. The sheaf embedding $\mathcal{G} \rightarrow \mathcal{L}\left(\mathcal{G}_{c}, \widetilde{\mathbb{C}}\right)$ is defined, for each open set $\Omega$ of $\mathbb{R}^{n}$, by the continuous map (as recalled in [17])

$$
\mathcal{G}(\Omega) \ni u \mapsto T_{u} \in \mathcal{L}\left(\mathcal{G}_{c}(\Omega), \widetilde{\mathbb{C}}\right),
$$


where $T_{u}$ is defined, for $u=\left[u_{\varepsilon}\right] \in \mathcal{G}(\Omega)$ and each $v=\left[v_{\varepsilon}\right] \in \mathcal{G}_{c}(\Omega)$, by

$$
\left\langle T_{u}, v\right\rangle=\left[\int_{K} u_{\varepsilon}(x) v_{\varepsilon}(x) d x\right] \in \widetilde{\mathbb{C}},
$$

where $K$ is an arbitrary compact set containing supp $v$ in its interior.

- $\mathcal{A}_{(\mathcal{C}, \mathcal{E}, \mathcal{P})}$ is a presheaf of $(\mathcal{C}, \mathcal{E}, \mathcal{P})$-algebras admitting $\mathcal{F}_{\mathcal{A}_{(\mathcal{C}, \mathcal{E}, \mathcal{P})}}$ as subpresheaf. We summarize the construction of the so-called $(\mathcal{C}, \mathcal{E}, \mathcal{P})$ algebras [14, 28, which generalize many cases met in the literature. $\mathbb{K}$ is the real or complex field and $\Lambda$ a set of indices. $\mathcal{C}$ is the factor ring $A / I$ where $I$ is an ideal of $A$, a given subring of $\mathbb{K}^{\Lambda}$. $(\mathcal{E}, \mathcal{P})$ is a sheaf of topological $\mathbb{K}$-algebras on a topological space $X$. A presheaf of $(\mathcal{C}, \mathcal{E}, \mathcal{P})$ algebras on $X$ is a presheaf $\mathcal{A}=\mathcal{H} / \mathcal{J}$ of factor algebras where $\mathcal{J}$ is an ideal of $\mathcal{H}$, a subsheaf of $\mathcal{E}^{\Lambda}$. The sections of $\mathcal{H}$ (resp. $\mathcal{J}$ ) of $X$ have to verify some estimates given by means of $\mathcal{P}$ and $A$ (resp. $I$ ). The above construction needs some technical conditions given in [14] on the structure of $\mathcal{C}$ and we suppose that for any open set $\Omega$ in $X$, the algebra $\mathcal{E}(\Omega)$ is endowed with a family $\mathcal{P}(\Omega)=\left(p_{i}\right)_{i \in I(\Omega)}$ of semi-norms. Then, the factors we speak about are given by

$$
\mathcal{H}(\Omega)=\mathcal{H}_{(A, \mathcal{E}, \mathcal{P})}(\Omega)=\left\{\left(u_{\lambda}\right)_{\lambda} \in[\mathcal{E}(\Omega)]^{\Lambda}: \forall i \in I(\Omega) \quad\left(\left(p_{i}\left(u_{\lambda}\right)\right)_{\lambda} \in|A|\right\}\right.
$$

and

$$
\mathcal{J}(\Omega)=\mathcal{J}_{\left(I_{A}, \mathcal{E}, \mathcal{P}\right)}(\Omega)=\left\{\left(u_{\lambda}\right)_{\lambda} \in[\mathcal{E}(\Omega)]^{\Lambda}: \forall i \in I(\Omega) \quad\left(p_{i}\left(u_{\lambda}\right)\right)_{\lambda} \in\left|I_{A}\right|\right\} .
$$

We assume further that $A$ is unitary and $\Lambda$ is left-filtering for the given (partial) order relation $\prec$. Let us denote by $\mathcal{F}$ a given sheaf of topological $\mathbb{K}$-vector spaces (resp. $\mathbb{K}$-algebras) over $X$ containing $\mathcal{E}$ as a subsheaf, and set

$$
\mathcal{F}_{\mathcal{A}}(\Omega)=\left\{u \in \mathcal{A}(\Omega): \exists\left(u_{\lambda}\right)_{\lambda} \in u \quad \exists f \in \mathcal{F}(\Omega) \lim _{\Lambda_{\mathcal{F}(\Omega)}} u_{\lambda}=f\right\} .
$$

Moreover, $\mathcal{F}_{\mathcal{A}}$ is a subpresheaf of vector spaces (resp. algebras) of $\mathcal{A}$. Roughly speaking, it is the presheaf whose sections above some open set $\Omega$ are the generalized functions in $\mathcal{A}(\Omega)$ associated to an element of $\mathcal{F}(\Omega)$. We can directly deduce the algebraic properties of $\mathcal{S}_{\mathcal{A}}^{\mathcal{F}_{\mathcal{A}}}(u)$ (see [14]) from Subsection 2.2. For the differential ones we suppose that $\mathcal{F}$ is a sheaf of topological differential vector spaces, with continuous differentiation, admitting $\mathcal{E}$ as a subsheaf of topological differential algebras. Then the presheaf $\mathcal{A}$ is also a presheaf of differential algebras.

3.4. $\mathcal{B}$-compatibility of differential or pseudo-differential operators. In all the previous examples, $\mathcal{B}$ is always a presheaf (or a sheaf) of differential algebras and $\mathcal{A}$ is a differential $\mathcal{B}$-module with a differentiation $\partial^{\alpha}\left(\alpha \in \mathfrak{A}=\mathbb{N}^{n}\right)$ extending the $\mathcal{B}$ one. Then in each case and each open set $V$ in $\Omega$ (open set of $X=\mathbb{R}^{n}$ ) it is easy to prove that $\partial^{\alpha} v$ maps $\mathcal{B}(V)$ into itself. Thus $\partial^{\alpha}$ is a presheaf operator $\mathcal{B}$-compatible in $\mathcal{A}(\Omega)$ according to Definition 3.9 . If we give now a family $\left(b_{\alpha}\right)_{\alpha \in \mathbb{N}^{n}}$ of elements in $\mathcal{B}(\Omega)$, then $P(\partial)=\sum_{|\alpha| \leq m} b_{\alpha} \partial^{\alpha}$ is a $\mathcal{B}$-compatible operator in $\mathcal{A}(\Omega)$.

Moreover at least in some cases, when $\mathcal{A}$ is $\mathcal{D}^{\prime}\left(\operatorname{resp} . \mathcal{G}, \mathcal{L}\left(\mathcal{G}_{c}(\Omega), \widetilde{\mathbb{C}}\right)\right.$ ), a pseudodifferential operator $A$ can be defined (see $[22]$ ) in $\mathcal{A}(\Omega)$ by the formula

$$
A u(x)=\iint_{\mathbb{R}^{n}} e^{i\langle(x-y), \xi\rangle} a(x, y, \xi) u(y) d y d \xi
$$


when $a(x, y, \xi)$ lies in the space $S_{\rho, \delta}^{m}\left(\Omega \times \Omega \times \mathbb{R}^{n}\right)$ of Hörmander symbols of order $m$ and type $(\rho, \delta)$. A extends continuously to a map $\mathcal{E}^{\prime}(\Omega) \rightarrow \mathcal{D}^{\prime}(\Omega)$. We can find (for example in [11]) a definition of generalized pseudo-differential operators with generalized symbols extending the classical one. The set $\widetilde{\mathcal{S}}_{\rho, \delta}^{m}\left(\Omega \times \mathbb{R}^{n}\right)$ of generalized symbols can be described (see [17]) as the algebra $\mathcal{G}_{\mathcal{S}_{\rho, \delta}^{m}}\left(\Omega \times \mathbb{R}^{n}\right)$ based on $\mathcal{S}_{\rho, \delta}^{m}\left(\Omega \times \mathbb{R}^{n}\right)$ and obtained as a $\mathcal{G}_{E}$-module by choosing $E=\mathcal{S}_{\rho, \delta}^{m}\left(\Omega \times \mathbb{R}^{n}\right)$. Then the pseudo-differential operator with generalized symbol $b \in \widetilde{\mathcal{S}}_{\rho, \delta}^{m}\left(\Omega \times \mathbb{R}^{n}\right)$ is the $\operatorname{map} \mathcal{G}_{c}(\Omega) \rightarrow \mathcal{G}(\Omega)$ given by

$$
A u=\int_{\mathbb{R}^{n}} e^{i x \xi} b(x, \xi) \widehat{u}(\xi) d \xi=\left[\int_{\mathbb{R}^{n}} e^{i x \xi} b_{\varepsilon}(x, \xi) \widehat{u}_{\varepsilon}(\xi) d \xi\right] .
$$

One can define more generally pseudo-differential operators by means of symbols in $\widetilde{\mathcal{S}}_{\rho, \delta}^{m}\left(\Omega \times \Omega \times \mathbb{R}^{n}\right)$ and generalized oscillatory integrals (see [15]). We can find in [17] (def. $2.5)$ an extension of the action of $A$ to the dual $\mathcal{L}\left(\mathcal{G}_{c}(\Omega), \widetilde{\mathbb{C}}\right)$.

When $\mathcal{A}$ is $\mathcal{D}^{\prime}$ (resp. $\left.\mathcal{G}, \mathcal{L}\left(\mathcal{G}_{c}(\Omega), \widetilde{\mathbb{C}}\right)\right)$, it is proved in each case that if $A$ is a properly supported pseudo-differential operator, it maps $\mathcal{A}(\Omega)$ into itself. Moreover, when $\mathcal{B}$ is $\mathrm{C}^{\infty}$, (resp. $\mathcal{G}^{\infty}, \mathcal{G}$ ), for each open set $V$ in $\Omega, A$ maps $\mathcal{B}(V)$ into itself. In other words $A$ is a $\mathcal{B}$-compatible operator in $\mathcal{A}(\Omega)$. Therefore Proposition 3.8 allows to deduce the classical inclusions:

$$
\mathcal{S}_{\mathcal{A}}^{\mathcal{B}}(P(\partial) u) \subset \mathcal{S}_{\mathcal{A}}^{B}(u) ; \quad \mathcal{S}_{\mathcal{A}}^{\mathcal{B}}(A u) \subset \mathcal{S}_{\mathcal{A}}^{B}(u),
$$

from the presheaf property of an operator $\mathcal{B}$-compatible. Through Proposition 3.11, we can even obtain some non linear results, when $\mathcal{A}$ is a presheaf of algebras as

$$
\mathcal{S}_{\mathcal{A}}^{\mathcal{B}}\left(\sum_{|\alpha| \leq m} b_{\alpha}\left(\partial^{\alpha} u\right)^{p_{\alpha}}\right) \subset \mathcal{S}_{\mathcal{A}}^{B}(u) ; \quad \mathcal{S}_{\mathcal{A}}^{\mathcal{B}}(A u)^{p} \subset \mathcal{S}_{\mathcal{A}}^{B}(u),
$$

where $p$ is any positive integer and $\left(p_{\alpha}\right)_{\alpha \in \mathbb{N}^{n}}$ any given family of positive integers.

\section{The frequential microlocal analysis}

\subsection{The distribution case}

4.1.1. Wave front set and microlocal regularity of product. For a general $v \in \mathcal{E}^{\prime}\left(\mathbb{R}^{n}\right)$ Hörmander introduces the cone $\Sigma(v)$ of all $\eta \in \mathbb{R}^{n} \backslash\{0\}$ having no conic neighbourhood $V$ such that the Fourier transform $\widehat{v}$ is rapidly decreasing in $V$. Lemma 8.1.1. in 21] proves that if $\Phi \in \mathcal{D}\left(\mathbb{R}^{n}\right)$ and $v \in \mathcal{E}^{\prime}\left(\mathbb{R}^{n}\right)$ then $\Sigma(\Phi v) \subset \Sigma(v)$. It follows that if $\Omega$ is an open set in $\mathbb{R}^{n}$ and $u \in \mathcal{D}^{\prime}(\Omega)$, setting: $\Sigma_{x}(u)=\bigcap_{\Phi} \Sigma(\Phi u) ; \Phi \in \mathcal{D}(\Omega), \Phi(x) \neq 0$, one can define the wave front set of $u$ as

$$
W F(u)=\left\{(x, \xi) \in \Omega \times\left(\mathbb{R}^{n} \backslash\{0\}\right): \xi \in \Sigma_{x}(u)\right\} .
$$

Then, if $u \in \mathcal{D}^{\prime}(\Omega)$ and $(x, \xi) \in \Omega \times\left(\mathbb{R}^{n} \backslash\{0\}\right), u$ is said to be in $H_{(s)}^{\text {loc }}$ at $(x, \xi)$ if $(x, \xi) \notin W F(u-v)$ for some $v \in H_{(s)}\left(\mathbb{R}^{n}\right)$. The microlocal regularity of products is given in the following result (see [23, 8.3.3):

TheOREM 4.1. Let $u_{j} \in H_{\left(s_{j}\right)}\left(\mathbb{R}^{n}\right), j=1,2$. Then

(i) $u_{1} u_{2} \in H_{\left(s_{2}\right)}^{\text {loc }}$ outside $W F\left(u_{1}\right)$, if $s_{1}>n / 2$ and $s_{1}+s_{2}>n / 2$;

(ii) $u_{1} u_{2} \in H_{(s)}^{\text {loc }}$ outside $W F\left(u_{1}\right)$, if $s_{1}<n / 2$ and $s_{1}+s_{2}-n / 2>s \geq 0$; 


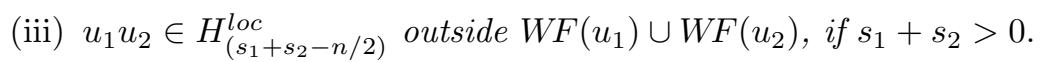

The propagation of noncharacteristic regularity for semi-linear equations studied in [35] is given by (see [23], 8.4.13):

THEOREM 4.2. Let $u \in H_{(s+k)}^{\text {loc }}(\Omega)$, where $\Omega$ is an open set in $R^{n}$ and $s>n / 2$, be a solution of the semi-linear equation

$$
P(x, D) u=f\left(x, J_{k} u\right),
$$

where $J_{k} u=\left(\partial^{\alpha} u\right)_{|\alpha \leq k|}, f$ and the coefficients of $P$ are $C^{\infty}$ and $k$ is smaller than the order $m$ of $P(x, D)$. If $P$ is noncharacteristic at $(x, \xi) \in \Omega \times\left(\mathbb{R}^{n} \backslash\{0\}\right)$, it follows that $u \in H_{(2 s+m-n / 2)}^{l o c}$ at $(x, \xi)$.

4.1.2. Application of paradifferential calculus. The paradifferential calculus of Bony 4] is based on some regularization of nonsmooth symbols. We do not intend to develop this theory here but look at it as a powerful tool to prove good results for nonlinear problems such as the following (see [23], 10.3.6):

THEOREM 4.3. Let $u \in H_{(s+m-1 / 2)}^{\text {loc }}(\Omega), s>\max ((n-1) / 2, n / 4)$, and assume that $u$ satisfies the quasilinear differential equation

$$
\sum_{|\alpha|=m} a_{\alpha}\left(x, J_{m-1} u(x)\right) \partial^{\alpha} u+c\left(x, J_{m-1} u(x)\right)=0,
$$

where $a_{\alpha}$ and $c$ are $C^{\infty}$. Then it follows that $u \in H_{(2 s+m-n / 2)}^{l o c}$ at every noncharacteristic point $(x, \xi)$.

4.1.3. Propagation of singularities. Roughly speaking we know that under some conditions for linear or pseudo-differential equations, the singularities of solutions propagate along bicharacteristics. This remains valid for nonlinear equations in the sense of the following (see [23], 11.4.1):

THEOREM 4.4. Let $u \in H_{(s+m)}^{l o c}(\Omega), s>n / 2+1$, be a real valued solution of the differential equation

$$
F\left(x, J_{m} u(x)\right)=0,
$$

where $F \in \mathrm{C}^{\infty}$. If $\sigma \leq 2 s-n / 2$, then the set of $(x, \eta) \in \Omega \times\left(\mathbb{R}^{n} \backslash\{0\}\right)$, where $u \notin H_{(\sigma+m-1)}^{\text {loc }}$ is contained in the characteristic set and it is invariant under the Hamilton flow defined by the principal symbol of the linearized equation.

Beals [2, 3] has studied the case of second order hyperbolic equations (extended by [5] to arbitrary order) for which we can give the following special version (see [23], 11.5.10): THEOREM 4.5. Let $u \in H_{(s)}^{\text {loc }}(\Omega), s>n / 2$, be a solution of the hyperbolic second order semi-linear equation

$$
P(x, \partial)=f(x, u)
$$

where $f \in \mathrm{C}^{\infty}$. If $u \in H_{(s)}^{\text {loc }}$ at a characteristic point $(x, \xi)$ and if $s \leq \sigma<3 s-n+1$, it follows that $u \in H_{(s)}^{\text {loc }}$ at the bicharacteristic $\gamma$ through $(x, \xi)$. 
4.2. The frequential microlocal analysis in $\mathcal{G}^{r}$. We refer the reader to [12, 13, for more details. The motivation is to summarize the microlocal results obtained for $\mathcal{A}=\mathcal{G}$ and $\mathcal{B}=\mathcal{G}^{\infty}$ (see [11, 31]) or $\mathcal{G}^{\mathcal{R}}$ (see [12]) into the microlocal ones involving $\mathcal{A}=\mathcal{G}^{r}$ and $\mathcal{B}=\mathcal{G}^{r, \mathcal{R}}$ (see [13]). On the other hand, we also summarize the microlocal studies for $\mathcal{A}=\mathcal{G}^{\sigma}$ and $\mathcal{B}=\mathcal{G}^{\sigma, \infty}$ (see [6]) or $\mathcal{A}=\mathcal{G}$ and $\mathcal{B}=\mathcal{G}^{A}$ (see [34]) or $\mathcal{G}^{L}$ (see [29]) into the microlocal ones involving $\mathcal{A}=\mathcal{G}^{r}$ and $\mathcal{B}=\mathcal{G}^{r, \mathcal{R}, L}$. We recall that $\mathcal{G}^{r}$ is a sheaf of algebras similar to the Colombeau one but constructed with an asymptotic scale $r=\left(r_{\lambda}\right)_{\lambda} \in\left(\mathbb{R}_{+}^{*}\right)^{\Lambda}$ such that $\lim _{\Lambda} r_{\lambda}=0$ and we specify below the construction of $\mathcal{G}^{r, \mathcal{R}}$ and $\mathcal{G}^{r, \mathcal{R}, L}$.

- The $\mathcal{G}^{r, \mathcal{R}}=\mathcal{X}^{r, \mathcal{R}} / \mathcal{N}^{r, \mathcal{R}}$ subsheaf of $\mathcal{G}^{r}$ is constructed from any asymptotic scale $r=\left(r_{\lambda}\right)_{\lambda} \in\left(\mathbb{R}_{+}^{*}\right)^{\Lambda}$ and any regular subset $\mathcal{R}$ of $\mathbb{R}_{+}^{\mathbb{N}}$ by

$$
\begin{aligned}
\mathcal{X}^{r, \mathcal{R}}(\Omega)=\left\{\left(u_{\lambda}\right)_{\lambda} \in\left[\mathrm{C}^{\infty}(\Omega)\right]^{\Lambda}:\right. & \forall K \Subset \Omega \exists N \in \mathcal{R} \\
& \left.\forall \alpha \in \mathbb{N}^{n} p_{K, \alpha}\left(u_{\lambda}\right)=O\left(r_{\lambda}^{-N(|\alpha|)}\right)\left(r_{\lambda} \rightarrow 0\right)\right\} ; \\
\mathcal{N}^{r, \mathcal{R}}(\Omega)=\left\{\left(u_{\lambda}\right)_{\lambda} \in\left[\mathrm{C}^{\infty}(\Omega)\right]^{\Lambda}:\right. & \forall K \Subset \Omega \forall m \in \mathcal{R} \\
& \left.\forall \alpha \in \mathbb{N}^{n} p_{K, \alpha}\left(u_{\lambda}\right)=O\left(r_{\lambda}^{m(|\alpha|)}\right)\left(r_{\lambda} \rightarrow 0\right)\right\} .
\end{aligned}
$$

- The $\mathcal{G}^{r, \mathcal{R}, L}=\mathcal{X}^{r, \mathcal{R}, L} / \mathcal{N}^{r, \mathcal{R}, L}$ subpresheaf of $\mathcal{G}^{r}$ is obtained when adding to the previous construction any $L=\left(L_{k}\right)$ sequence specified in Subsection 2.3:

$$
\begin{gathered}
\mathcal{X}^{r, \mathcal{R}, L}(\Omega)=\left\{\left(u_{\lambda}\right)_{\lambda} \in\left[\mathrm{C}^{\infty}(\Omega)\right]^{\Lambda}: \forall K \Subset \Omega \exists N \in \mathcal{R} \exists c>0 \exists \lambda_{0} \in \Lambda\right. \\
\left.\forall \alpha \in \mathbb{N}^{n} \forall \lambda \prec \lambda_{0} \sup _{x \in K}\left|D^{\alpha} u_{\lambda}(x)\right| \leq c r_{\lambda}^{-N(|\alpha|)}\left(c L_{|\alpha|}\right)^{|\alpha|}\right\} ; \\
\mathcal{N}^{r, \mathcal{R}, L}(\Omega)=\mathcal{X}^{r, \mathcal{R}, L}(\Omega) \cap \mathcal{N}^{r}(\Omega) .
\end{gathered}
$$

- The microlocal studies in $\mathcal{G}^{r}$ are based on the "rough" rapidly decreasing functions of the space

$$
\mathcal{S}_{*}(\Omega)=\left\{f \in \mathrm{C}^{\infty}(\Omega): \forall q \in \mathbb{N} \mu_{q, 0}(f)<+\infty\right\},
$$

with $\mu_{q, \alpha}(f)=\sup _{x \in \Omega}(1+|x|)^{q}\left|\partial^{\alpha} f(x)\right|$; we have, for $u \in \mathcal{E}^{\prime}\left(\mathbb{R}^{n}\right)$,

$$
u \in \mathrm{C}^{\infty}\left(\mathbb{R}^{n}\right) \Leftrightarrow \mathcal{F}(u) \in \mathcal{S}_{*}\left(\mathbb{R}^{n}\right) .
$$

- The algebra $\mathcal{G}_{\mathcal{S}_{*}}^{r, \mathcal{R}}(\Omega)=\mathcal{X}_{\mathcal{S}_{*}}^{r, \mathcal{R}}(\Omega) / \mathcal{N}_{\mathcal{S}_{*}}^{r}(\Omega)$ is constructed in the same way as previously (when $\mathcal{R}$ is a regular subset of $\mathbb{R}_{+}^{\mathbb{N}}$ and $\Omega$ an open subset of $\mathbb{R}^{n}$ ) from

$$
\begin{gathered}
\mathcal{X}_{\mathcal{S}_{*}^{r}}^{r, \mathcal{R}}(\Omega)=\left\{\left(f_{\varepsilon}\right)_{\varepsilon} \in \mathcal{S}_{*}(\Omega)^{\Lambda}: \exists N \in \mathcal{R} \forall q \in \mathbb{N} \mu_{q, 0}\left(f_{\varepsilon}\right)=\mathrm{O}\left(r_{\lambda}^{-N(q)}\right) \text { as } \lambda \rightarrow 0\right\} ; \\
\mathcal{N}_{\mathcal{S}_{*}}^{r}(\Omega)=\left\{\left(f_{\varepsilon}\right)_{\varepsilon} \in \mathcal{S}_{*}(\Omega)^{\Lambda}: \forall N \in \mathbb{R}_{+}^{\mathbb{N}} \forall q \in \mathbb{N} \mu_{q, 0}\left(f_{\varepsilon}\right)=\mathrm{O}\left(r_{\lambda}^{N(q)}\right) \text { as } \lambda \rightarrow 0\right\} .
\end{gathered}
$$

4.2.1. The $\mathcal{G}^{r, \mathcal{R}}$-generalized wave front set

Definition 4.6. An element $u \in \mathcal{G}^{r}(\Omega)$ is said microlocally $(r, \mathcal{R})$-regular at $\left(x_{0}, \xi_{0}\right) \in$ $\Omega \times\left(\mathbb{R}^{d} \backslash\{0\}\right)$ (we set: $\left.u \in \mathcal{G}^{r, \mathcal{R}}\left(x_{0}, \xi_{0}\right)\right)$ if there exists some neighbourhood $W$ of $x_{0}$, some $\varphi \in \mathcal{D}(W), \varphi\left(x_{0}\right) \neq 0$, some conic neighbourhood $\Gamma$ of $\xi_{0}$ such that $\widehat{\varphi u} \in \mathcal{G}_{s_{*}}^{r, \mathcal{R}}(\Gamma)$.

Definition 4.7. The $\mathcal{G}^{r, \mathcal{R}}$-generalized wave front set $W F^{(r, \mathcal{R})}(u)$ of $u \in \mathcal{G}^{r}(\Omega)$ is the complement in $\Omega \times\left(\mathbb{R}^{n} \backslash\{0\}\right)$ of the set of all pairs $\left(x_{0}, \xi_{0}\right)$ such that $u$ is microlocally $(r, \mathcal{R})$-regular at $\left(x_{0}, \xi_{0}\right)$.

THEOREM 4.8. The projection of $W F^{(r, \mathcal{R})}(u)$ in $\Omega$ is equal to $\operatorname{sing} \operatorname{supp}^{(r, \mathcal{R})}(u)$. 
The proof follows the arguments involved in 21] using lemma 8.1.1.

EXAmPLE 4.9. Taking $\lambda=\varepsilon \in] 0,1], r_{\varepsilon}=\varepsilon$ and $\mathcal{R}=\mathcal{B} o$ (the set of bounded sequences), we obtain the $\mathcal{G}^{\infty}$ microlocal analysis of elements in $\mathcal{G}$ (see [31, 37]).

Taking $\lambda=\varepsilon \in] 0,1]$ and $r_{\varepsilon}=\varepsilon$, we obtain for any $\mathcal{R}$ the $\mathcal{G}^{\mathcal{R}}$ microlocal analysis of elements in $\mathcal{G}$ (see [12]).

4.2.2. The $\mathcal{G}^{r, \mathcal{R}, L}$-generalized wave front set. We begin by giving a characterization of $\mathcal{G}^{r, \mathcal{R}, L}$-local regularity. When starting from previous cases (like $\mathcal{G}, \mathcal{G}^{\mathcal{R}}$ or $\mathcal{G}^{L}$ ) the problem is to change simultaneously the asymptotic scale into a new one, and the $\mathcal{G}^{\infty}$-regularity subordinated to $L$-conditions into $\mathcal{G}^{\mathcal{R}}$-regularity subordinated to $L$-conditions. To do that we have to mix carefully the techniques used in [12] and [29]. This study is done in [13]. In this subsection, we only give the definitions and results without proofs.

THEOREM 4.10. Let $x_{0} \in \Omega \subset R^{n}$ and $u \in G^{r}(\Omega)$. Then, $u$ is $G^{r, \mathcal{R}, L}$ at $x_{0}$ (in the sense of Definition 3.2) iff there exist some neighbourhood $W$ of $x_{0}$, a compact $K$ such $W \subset K \Subset \Omega$, a sequence of functions $\chi_{k}$, each in $\mathcal{D}_{K}(\Omega)$ and valued in $[0,1]$ with $\chi_{k} u=u$ on $W$, a representative $\left(u_{\lambda}\right)_{\lambda}$ of $u$, a regular sequence $N \in R$, a positive constant $c$, and $\lambda_{0} \in \Lambda$ such that for all $\xi \in R$

$$
\forall k \in \mathbb{N} \forall \lambda \prec \lambda_{0} \quad|\xi|^{k}\left|\widehat{u_{k, \lambda}}(\xi)\right| \leq c r_{\lambda}^{-N(k)}\left(c L_{k}\right)^{k} .
$$

Definition 4.11. An element $u \in \mathcal{G}^{r}(\Omega)$ is said to be microlocally $(r, \mathcal{R}, L)$-regular at $\left(x_{0}, \xi_{0}\right) \in \Omega \times\left(\mathbb{R}^{n} \backslash\{0\}\right)$ (we write $\left.u \in \mathcal{G}^{r, \mathcal{R}, L}\left(x_{0}, \xi_{0}\right)\right)$ if there exist a neighbourhood $W$ of $x_{0}$, a conic neighbourhood $\Gamma$ of $\xi_{0}$, a sequence $\left(u_{k}\right)_{k \in \mathbb{N}}$ of generalized functions, where $u_{k}=\chi_{k} u$ and each $\chi_{k}$ is valued in $[0,1]$ and is in $\mathcal{D}_{K}(\Omega)$, with $W \subset K \Subset \Omega, u_{k}$ being equal to $u$ in $W$, a sequence $N \in \mathcal{R}$, a positive constant $c$, and $\lambda_{0} \in \Lambda$ such that $(*$ holds when $\xi \in \Gamma$.

Definition 4.12. The $\mathcal{G}^{r, \mathcal{R}, L}$-generalized wave front set $W F^{(r, \mathcal{R}, L)}(u)$ of $u \in \mathcal{G}^{r}(\Omega)$ is the complement in $\Omega \times\left(\mathbb{R}^{n} \backslash\{0\}\right)$ of the set of all pairs $\left(x_{0}, \xi_{0}\right)$ such that $u$ is microlocally $(r, \mathcal{R}, L)$-regular at $\left(x_{0}, \xi_{0}\right)$.

Clearly, $W F^{r, \mathcal{R}, L}(u)$ is a closed subset of $\Omega \times\left(\mathbb{R}^{n} \backslash\{0\}\right)$; its projection in $\Omega$ is given by the following result:

THEOREM 4.13. The projection of $W F^{(r, \mathcal{R}, L)}(u)$ in $\Omega$ is equal to $\operatorname{sing} \operatorname{supp}^{(r, \mathcal{R}, L)}(u)$.

EXAmPLE 4.14. Taking $\lambda=\varepsilon \in] 0,1], r_{\varepsilon}=\varepsilon, \mathcal{R}=\mathcal{B} o$ (the set of bounded sequences), we obtain for any $L$ the $\mathcal{G}^{L}$ microlocal analysis of elements in $\mathcal{G}$ (see [29]).

Taking $\lambda=\varepsilon \in] 0,1], r_{\varepsilon}=\varepsilon, \mathcal{R}=\mathcal{B} o$ and $L_{k}=k+1$, we get the $\mathcal{G}^{A}$ microlocal analysis of elements in $\mathcal{G}$ (see 34 ).

Taking $\lambda=\varepsilon \in] 0,1], r_{\varepsilon}=e^{\varepsilon^{-\frac{1}{2 s-1}}}, \mathcal{R}=\mathcal{B} o$ and $L_{k}=(k+1)^{s}$, we obtain the $\mathcal{G}^{s, \infty}$ micolocal analysis of elements in $\mathcal{G}^{s}$ (see [6]).

4.2.3. Propagation of singularities under differential (or pseudo-differential) operators. We distinguish two cases.

a) Summarizing the first investigations in the following results proved in [13. we have Proposition 4.15. Suppose that $(a, u)$ is in $G^{r}(\Omega) \times G^{r}(\Omega)$. 
(i) If $a \in G^{r, \mathcal{R}}(\Omega) \quad$ (resp. $a \in G^{r, \mathcal{R}, L}(\Omega)$ ), then $W F^{(r, \mathcal{R})}(a u) \subset W F^{(r, \mathcal{R})}(u)$ (resp. $\left.W F^{(r, \mathcal{R}, L)}(a u) \subset W F^{(r, \mathcal{R}, L)}(u)\right)$;

(ii) $W F^{(r, \mathcal{R})}\left(\partial^{\alpha} u\right) \subset W F^{(r, \mathcal{R})}(u)$ and $W F^{(r, \mathcal{R}, L)}\left(\partial^{\alpha} u\right) \subset W F^{(r, \mathcal{R}, L)}(u)$.

Proposition 4.16. Let $P(\partial)=\sum_{|\alpha| \leq m} a_{\alpha} \partial^{\alpha}$ be a differential operator in $G^{r}(\Omega)$. If the coefficients $a_{\alpha}$ lie in $G^{r, \mathcal{R}}(\Omega)$ (resp. in $G^{r, \mathcal{R}, L}(\Omega)$ ), then

$$
W F^{(r, \mathcal{R})}(P(\partial) u) \subset W F^{(r, \mathcal{R})}(u)\left(\operatorname{resp} . W F^{(r, \mathcal{R}, L)}(P(\partial) u) \subset W F^{(r, \mathcal{R}, L)}(u)\right) .
$$

b) In the special case of $\mathcal{G}^{\infty}$ singularities of $\mathcal{G}$, we can quote the results based on pseudo-differential operators and pseudo-differential techniques. In [18] analogues of Hörmander definition of the distributional wave front set given in [22] are obtained by characterizations of generalized wave front set in terms of intersection over some nonellipticity domains. This intersection is taken over all slow scale pseudo-differential operators $a \in \widetilde{\mathcal{S}}_{s c}^{m}\left(\Omega \times \mathbb{R}^{n}\right)$ (Definition 1.1) verifying some other regularity conditions. More precisely, if $E l l_{s c}(a)$ denotes the set of all $(x, \xi) \in \Omega \times T^{*}(\Omega) \backslash\{0\}$, where $a$ is slow scale micro-elliptic (Definition 1.2), Theorem 2.1 proves that, for all $u \in G(\Omega)$,

$$
W F_{g}(u)=W F_{s c}(u):=\bigcap_{\substack{a(x, D) \in \in_{p r} \Psi^{0}(\Omega) \\ a(x, D) u \in \mathcal{G}^{\infty}(\Omega)}} E l l_{s c}(a)^{c}
$$

where ${ }_{p r} \Psi^{0}(\Omega)$ denotes the set of all properly supported slow scale operators of order 0 .

\subsection{The frequential microlocal analysis in $\mathcal{L}\left(\mathcal{G}_{c}(\Omega), \widetilde{\mathbb{C}}\right)$}

4.3.1. The generalized wave front set $W F_{\mathcal{G}}(T)$. Garetto in [17. (Definition 3.3) defines the $\mathcal{G}$-wave front set of a functional $T \in \mathcal{L}\left(\mathcal{G}_{c}(\Omega), \widetilde{\mathbb{C}}\right)$ as

$$
W F_{\mathcal{G}}(T):=\bigcap_{\substack{a(x, D) \in \in_{p r} \Psi^{0}(\Omega) \\ a(x, D) T \in \mathcal{G}(\Omega)}} \operatorname{Ell}_{s c}(a)^{c} .
$$

And even the $\mathcal{G}^{\infty}$-wave front set of $T$ is defined in the same way by replacing $\mathcal{G}(\Omega)$ by $\mathcal{G}^{\infty}(\Omega)$. Proposition 3.5 shows that the projection on $\Omega$ of $W F_{\mathcal{G}}(T)$ is exactly $\operatorname{sing} \operatorname{supp}_{\mathcal{G}} T$. When $A$ is a properly supported pseudo-differential operator with symbol $a \in \widetilde{\mathcal{S}}_{\rho, \delta}^{m}(\Omega \times$ $\left.\mathbb{R}^{n}\right)$, the inclusion

$$
W F_{\mathcal{G}}(A T) \subset W F_{\mathcal{G}}(T)
$$

can be refined by introducing the concept of $\mathcal{G}$-microsupport of $a$, denoted $\mu \operatorname{supp}_{\mathcal{G}}(a)$. It is the complement of all $(x, \xi) \in \Omega \times T^{*}(\Omega) \backslash\{0\}$ where $a$ is $\mathcal{G}$-smoothing (Definition 3.6). Then we have (Corollary 3.9)

$$
W F_{\mathcal{G}}(a(x, D) T) \subset W F_{\mathcal{G}}(T) \cap \mu \operatorname{supp}_{\mathcal{G}}(a) .
$$

4.3.2. Fourier transform characterization of $T$ and propagation of singularities. When $\varphi \in \mathcal{D}(\Omega)$ and $T \in \mathcal{D}^{\prime}(\Omega)$, we recall that the regularity of $\varphi T$ can be measured by the rapid decay of its Fourier transform in some conic region $\Gamma \subset \mathbb{R}^{n} \backslash\{0\}$. Following this idea, Garetto introduces the subset $\mathcal{G}_{\mathcal{S}, 0}(\Gamma)$ of $\mathcal{G}_{\tau}\left(\mathbb{R}^{n}\right)$ (algebra of tempered generalized functions) as follows:

$$
\mathcal{G}_{\mathcal{S}, 0}(\Gamma):=\left\{u=\left[u_{\varepsilon}\right] \in \mathcal{G}_{\tau}\left(\mathbb{R}^{n}\right): \forall l \in \mathbb{R} \exists N \in \mathbb{N} \sup _{x \in \Gamma}(1+|x|)^{l}\left|u_{\varepsilon}(x)\right|=O\left(\varepsilon^{-N}\right)\right\}
$$


which is similar to $\mathcal{G}_{s_{*}}^{r, \mathcal{R}}(\Gamma)$ introduced in Subsection 3.2. This leads to the following Fourier transform characterization of $T$ (see [18], 3.15 or [17, 3.10):

TheOREM 4.17. Let $T$ be a basic functional in $\mathcal{L}\left(\mathcal{G}_{c}(\Omega), \widetilde{\mathbb{C}}\right)$. Then $(x, \xi) \notin W F_{\mathcal{G}}(T)$ if and only if there exist a conic neighbourhood of $\xi$ and a cutoff function $\Phi \in D(\Omega)$ with $\Phi(x)=1$ such that

$$
\mathcal{F}(\Phi T) \subset \mathcal{G}_{\mathcal{S}, 0}(\Gamma) .
$$

Then an extension of Theorem 4.1 follows (see [15], [17, 4.1):

THEOREM 4.18. If $A=a(x, D)$ is a properly supported pseudo-differential operator with symbol $a \in \underline{\widetilde{S}}_{s c}^{m}\left(\Omega \times \mathbb{R}^{n}\right)$ and $T$ a basic functional in $\mathcal{L}\left(\mathcal{G}_{c}(\Omega), \widetilde{\mathbb{C}}\right)$, then

$$
W F_{\mathcal{G}}(A T) \subset W F_{\mathcal{G}}(T) \subset W F_{\mathcal{G}}(A T) \cup E l l_{s c}(a)^{c} .
$$

5. The asymptotic microlocal analysis. Let $\Omega$ be an open set in $X$. Fix $u=\left[u_{\lambda}\right] \in$ $\mathcal{A}(\Omega)$ and $x \in \Omega$. The idea of the $(a, \mathcal{F})$-microlocal analysis is the following: $\left(u_{\lambda}\right)_{\lambda}$ may not tend to a section of $\mathcal{F}$ above a neighbourhood of $x$, that is, there may not exist $V \in \mathcal{V}_{x}$ and $f \in \mathcal{F}(V)$ such that $\lim _{\Lambda} \mathcal{F}(V) u_{\lambda}=f$. Nevertheless, in this case, there may exist $V \in \mathcal{V}_{x}, r \geq 0$ and $f \in \mathcal{F}(V)$ such that $\lim _{\Lambda} \mathcal{F}(V) a_{\lambda}(r) u_{\lambda}=f$, that is $\left[\left.a_{\lambda}(r) u_{\lambda}\right|_{V}\right]$ is in the subspace (resp. subalgebra) $\mathcal{F}_{\mathcal{A}}(V)$ of $\mathcal{A}(V)$ introduced in Subsection 2.5. These preliminary remarks lead to the following concept and results which we summarize from the results given in [27, 28, 14].

5.1. The $(a, \mathcal{F})$-singular parametric spectrum. We recall that $a$ is a map from $\mathbb{R}_{+}$ to $A_{+}$such that $a(0)=1$ and $\mathcal{F}$ is a presheaf of topological vector spaces (or topological algebras). For any open subset $\Omega$ of $X, u=\left[u_{\lambda}\right] \in \mathcal{A}(\Omega)$ and $x \in \Omega$, set

$$
\begin{aligned}
N_{(a, \mathcal{F}), x}(u) & =\left\{r \in \mathbb{R}_{+}: \exists V \in \mathcal{V}_{x} \quad \exists f \in \mathcal{F}(V) \quad \lim _{\Lambda} \mathcal{F}(V)\right. \\
& \left.=\left\{r \in \mathbb{R}_{+}:\left.\exists V \in \mathcal{V}_{x}(r) u_{\lambda} u_{\lambda}(r) u_{\lambda}\right|_{V}\right] \in \mathcal{F}_{\mathcal{A}}(V)\right\} .
\end{aligned}
$$

It is easy to check that $N_{(a, \mathcal{F}), x}(u)$ does not depend on the representative of $u$. If no confusion may arise, we shall simply write $N_{(a, \mathcal{F}), x}(u)=N_{x}(u)$.

Assume that

(a) $\forall \lambda \in \Lambda \quad \forall(r, s) \in \mathbb{R}_{+} a_{\lambda}(r+s) \leq a_{\lambda}(r) a_{\lambda}(s)$ and, for all $r \in \mathbb{R}_{+} \backslash\{0\}$, the net $\left(a_{\lambda}(r)\right)_{\lambda}$ converges to 0 in $\mathbb{K}$;

(b) $\mathcal{F}$ is a presheaf of Hausdorff locally convex topological vector spaces.

Then, from Theorem 7 in [14] we have, for $u \in \mathcal{A}(\Omega)$ :

(i) If $r \in N_{x}(u)$, then $[r,+\infty)$ is included in $N_{x}(u)$. Moreover, for all $s>r$, there exists $V \in \mathcal{V}_{x}$ such that: $\lim _{\Lambda} \mathcal{F}(V)\left(\left.a_{\lambda}(s) u_{\lambda}\right|_{V}\right)=0$. Consequently, $N_{x}(u)$ is either empty, or a sub-interval of $\mathbb{R}_{+}$.

(ii) More precisely, suppose that for $x \in \Omega$, there exist $r \in \mathbb{R}_{+}, V \in \mathcal{V}_{x}$ and $f \in \mathcal{F}(V)$, nonzero on each neighbourhood of $x$ included in $V$, such that

$$
\lim _{\Lambda} \mathcal{F ( V )}\left(\left.a_{\lambda}(r) u_{\lambda}\right|_{V}\right)=f .
$$

Then $N_{x}(u)=[r,+\infty)$. 
(iii) In the situation of $(i)$ and $(i i)$, we have: $0 \in N_{x}(u)$ iff $N_{x}(u)=\mathbb{R}_{+}$. Moreover, if one of these assertions holds, the limits $\lim _{\Lambda} \mathcal{F}(V)\left(\left.a_{\lambda}(s) u_{\lambda}\right|_{V}\right)$ can be nonnull only for $s=0$.

Now, we set

$$
\Sigma_{(a, \mathcal{F}), x}(u)=\Sigma_{x}(u)=\mathbb{R}_{+} \backslash N_{x}(u) ; \quad R_{(a, \mathcal{F}), x}(u)=R_{x}(u)=\inf N_{x}(u) .
$$

According to the previous remarks and comments, $\Sigma_{(a, \mathcal{F}), x}(u)$ is an interval of $\mathbb{R}_{+}$of the form $\left[0, R_{(a, \mathcal{F}), x}(u)\right)$ or $\left[0, R_{(a, \mathcal{F}), x}(u)\right]$, the empty set, or $\mathbb{R}_{+}$. This leads to the following (see [14]):

Definition 5.1. The $(a, \mathcal{F})$-singular spectrum of $u \in A(\Omega)$ is the set

$$
\mathcal{S}_{\mathcal{A}}^{(a, \mathcal{F})}(u)=\left\{(x, r) \in \Omega \times \mathbb{R}_{+}: r \in \Sigma_{x}(u)\right\} .
$$

ExAmple 5.2. Set $X=\mathbb{R}^{d}, \mathcal{E}=\mathrm{C}^{\infty}, \mathcal{F}=\mathrm{C}^{p}(p \in \overline{\mathbb{N}}=\mathbb{N} \cup\{+\infty\}), f \in \mathrm{C}^{\infty}(\Omega)$. Set $u=\left[\left(\varepsilon^{-1} f\right)_{\varepsilon}\right]$ and $v=\left[\left(\varepsilon^{-1}|\ln \varepsilon| f\right)_{\varepsilon}\right]$ in $\mathcal{A}(\Omega)=\mathcal{G}(\Omega)$. Then, for all $x \in \mathbb{R}$,

$$
N_{\left(a, \mathrm{C}^{p}\right), x}(u)=[1,+\infty), \quad N_{\left(a, \mathrm{C}^{p}\right), x}(v)=(1,+\infty), \quad R_{\left(a, \mathrm{C}^{p}\right), x}(u)=R_{\left(a, \mathrm{C}^{p}\right), x}(v)=1 .
$$

REMARK 5.3. We have $\Sigma_{(a, \mathcal{F}), x}(u)=\varnothing$ iff $N_{(a, \mathcal{F}), x}(u)=\mathbb{R}_{+}$and, according to Theorem 7 in [14, iff $0 \in N_{(a, \mathcal{F}), x}(u)$, that is, there exist $(V, f) \in V_{x} \times \mathcal{F}(V)$ such that $\lim _{\Lambda} \mathcal{F}(V)\left(\left.a_{\lambda}(0) u_{\lambda}\right|_{V}\right)=f$. As $a_{\lambda}(0) \equiv 1$, this last assertion is equivalent to $x \in \mathcal{O}_{\mathcal{A}}^{\mathcal{F}}(u)$. Thus $\Sigma_{(a, \mathcal{F}), x}(u)=\emptyset$ iff $x \notin \mathcal{S}_{\mathcal{A}}^{\mathcal{F}}(u)$.

Proposition 5.4. The projection of the $(a, \mathcal{F})$-singular spectrum of $u$ on $\Omega$ is the $\mathcal{F}$ singular support of $u$.

5.2. Some properties of the $(a, \mathcal{F})$-singular parametric spectrum. We begin with introducing the following notation. For $u=\left[u_{\lambda}\right] \in \mathcal{A},(\Omega)$, the symbol

$$
\lim _{\Lambda} \mathcal{F}(V)\left(\left.a_{\lambda}(r) u_{\lambda}\right|_{V}\right) \in \mathcal{F}(V)
$$

means that there exists $f \in \mathcal{F}(V)$ such that $\lim _{\Lambda} \mathcal{F}(V)\left(\left.a_{\lambda}(r) u_{\lambda}\right|_{V}\right)=f$.

5.2.1. Linear and differential properties. It is easy to prove that for any $u, v \in \mathcal{A}(\Omega)$, we have

$$
\mathcal{S}_{\mathcal{A}}^{(a, \mathcal{F})}(u+v) \subset \mathcal{S}_{\mathcal{A}}^{(a, \mathcal{F})}(u) \cup \mathcal{S}_{\mathcal{A}}^{(a, \mathcal{F})}(v)
$$

As a corollary, we have

$$
\mathcal{S}_{\mathcal{A}}^{(a, \mathcal{F})}(u)=\mathcal{S}_{\mathcal{A}}^{(a, \mathcal{F})}\left(u_{1}\right)
$$

for any $u, u_{0}, u_{1}$ in $\mathcal{A}(\Omega)$ such that

$$
\text { (i) } u=u_{0}+u_{1}, \quad(\text { ii }) \mathcal{S}_{\mathcal{A}}^{(a, \mathcal{F})}\left(u_{0}\right)=\varnothing .
$$

Assume that $\mathcal{F}$ is a sheaf of topological differential vector spaces, with continuous differentiation, admitting $\mathcal{E}$ as a subsheaf of topological differential algebras. Then the sheaf $\mathcal{A}$ is also a sheaf of differential algebras with $\partial^{\alpha} u=\left[\partial^{\alpha} u_{\lambda}\right]$ for any $\alpha \in \mathbb{N}^{d}$ and $u \in \mathcal{A}(\Omega)$, where $\left(u_{\lambda}\right)_{\lambda}$ is an arbitrary representative of $u$. The independence of $\partial^{\alpha} u$ from the choice of a representative follows directly from the definition of $\mathcal{J}_{\left(I_{A}, \mathcal{E}, \mathcal{P}\right)}$. It follows that if $u$ is in $\mathcal{A}(\Omega)$ and $C_{\alpha}$ in $\mathcal{E}(\Omega)$, then

$$
\mathcal{S}_{\mathcal{A}}^{(a, \mathcal{F})}(P(\partial) u) \subset \mathcal{S}_{\mathcal{A}}^{(a, \mathcal{F})}(u)
$$

where $P(\partial)=\sum_{|\alpha| \leq m} C_{\alpha} \partial^{\alpha}$ for $\alpha \in \mathbb{N}^{d}$. 
5.2.2. Nonlinear properties. If $\mathcal{F}$ is a presheaf of algebras, the $(a, \mathcal{F})$-singular spectrum inherits new properties with respect to nonlinear operations, as seen in the following results (see in [14]):

THEOREM 5.5. Suppose that $F$ is a presheaf of algebras. For $u, v \in A(\Omega)$ denote:

$$
D_{0}=\mathcal{S}_{\mathcal{A}}^{\mathcal{F}}(u) \cap \mathcal{S}_{\mathcal{A}}^{\mathcal{F}}(v) ; \quad D_{1}=\mathcal{S}_{\mathcal{A}}^{\mathcal{F}}(u) \backslash D_{0} ; \quad D_{2}=\mathcal{S}_{\mathcal{A}}^{\mathcal{F}}(v) \backslash D_{0} .
$$

Then the $(a, F)$-singular asymptotic spectrum of uv satisfies

$$
\begin{aligned}
\mathcal{S}_{\mathcal{A}}^{(a, \mathcal{F})}(u v) \subset & \left\{(x, \xi): x \in D_{1}, \xi \in \Sigma_{x}(u)\right\} \cup\left\{(x, \xi): x \in D_{2}, \xi \in \Sigma_{x}(v)\right\} \\
& \cup\left\{(x, \xi): x \in D_{0}, \xi \in E_{x}(u, v)\right\},
\end{aligned}
$$

where

$$
E_{x}(u, v)= \begin{cases}{\left[0, \sup \Sigma_{x}(u)+\sup \Sigma_{x}(v)\right],} & \text { if } \Sigma_{x}(u) \neq \mathbb{R}_{+} \text {and } \Sigma_{x}(v) \neq \mathbb{R}_{+} \\ \mathbb{R}_{+}, & \text {if } \Sigma_{x}(u)=\mathbb{R}_{+} \text {or } \Sigma_{x}(v)=\mathbb{R}_{+}\end{cases}
$$

for any $x \in D_{0}$.

COROLlary 5.6. If $F$ is a presheaf of topological algebras, then

$$
\mathcal{S}_{\mathcal{A}}^{(a, \mathcal{F})}\left(u^{p}\right) \subset\left\{\left(x, H_{p, x}(u)\right): x \in \mathcal{S}_{\mathcal{A}}^{\mathcal{F}}(u)\right\}
$$

for $u \in A(\Omega)$ and $p \in N^{*}$, where

$$
H_{p, x}(u)= \begin{cases}{\left[0, p \sup \Sigma_{x}(u)\right],} & \text { if } \Sigma_{x}(u) \neq \mathbb{R}_{+} \\ \mathbb{R}_{+}, & \text {if } \Sigma_{x}(u)=\mathbb{R}_{+}\end{cases}
$$

5.3. Some examples and applications to partial differential equations. In this subsection we shall give some examples of $(a, \mathcal{F})$-singular spectra of solutions to nonlinear partial differential equations given in ([14], Subsection 4.2). Throughout we shall suppose that $\Lambda=] 0,1], X=\mathbb{R}^{d}, \mathcal{E}=\mathrm{C}^{\infty}, \mathcal{F}=\mathrm{C}^{p}(1 \leq p \leq \infty)$ or $\mathcal{F}=\mathcal{D}^{\prime}, a_{\varepsilon}(r)=\varepsilon^{r}$. The results will hold for any $(\mathcal{C}, \mathcal{E}, \mathcal{P})$-algebra

$$
\mathcal{A}=\mathcal{H}_{(A, \mathcal{E}, \mathcal{P})} / \mathcal{J}_{\left(I_{A}, \mathcal{E}, \mathcal{P}\right)}
$$

such that $\left(a_{\varepsilon}(r)\right)_{\varepsilon} \in A_{+}$for all $r \in \mathbb{R}_{+}$and the hypothesis given in 2.6.2 holds.

5.3.1. On the singular spectrum of powers of the delta function. We can compare the $\left(a, \mathrm{C}^{p}\right)$-singular spectrum and the $\left(a, \mathcal{D}^{\prime}\right)$-singular spectrum of powers of the delta function. Given a mollifier of the form

$$
\varphi_{\varepsilon}(x)=\frac{1}{\varepsilon^{d}} \varphi\left(\frac{x}{\varepsilon}\right), \quad x \in \mathbb{R}^{d},
$$

where $\varphi \in \mathcal{D}\left(\mathbb{R}^{d}\right), \varphi \geq 0$ and $\int \varphi(x) d x=1$, its class in $\mathcal{A}\left(\mathbb{R}^{d}\right)$ defines the delta function $\delta(x)$ as an element of $\mathcal{A}\left(\mathbb{R}^{d}\right)$. Its powers are given by $(m \in \mathbb{N})$

$$
\delta^{m}=\left[\varphi_{\varepsilon}^{m}\right]=\left[\frac{1}{\varepsilon^{m d}} \varphi^{m}\left(\frac{\dot{\bar{\varepsilon}}}{\varepsilon}\right)\right] .
$$

Clearly, the $\mathrm{C}^{0}$-singular spectrum is given by

$$
\mathcal{S}_{\mathcal{A}}^{\left(a, \mathrm{C}^{0}\right)}\left(\delta^{m}\right)=(0,[0, m d]) .
$$


Differentiating $\varphi^{m}(x)$ and observing that for each derivative there is a point $x$ at which this function does not vanish we obtain the $\left(a, \mathrm{C}^{p}\right)$-singular spectrum of $\delta^{m}$ :

$$
\mathcal{S}_{\mathcal{A}}^{\left(a, \mathrm{C}^{p}\right)}\left(\delta^{m}\right)=(0,[0, m d+p]) .
$$

Given now a test function $\psi \in \mathcal{D}\left(\mathbb{R}^{d}\right)$, we have

$$
\int \varphi_{\varepsilon}^{m}(x) \psi(x) d x=\int \frac{1}{\varepsilon^{m d-d}} \varphi^{m}(x) \psi(\varepsilon x) d x,
$$

thus the $\left(a, D^{\prime}\right)$-singular spectrum of $\delta^{m}$ is

$$
\mathcal{S}_{\mathcal{A}}^{\left(a, \mathcal{D}^{\prime}\right)}\left(\delta^{m}\right)=\varnothing \text { for } m=1, \quad \mathcal{S}_{\mathcal{A}}^{\left(a, \mathcal{D}^{\prime}\right)}\left(\delta^{m}\right)=(0,[0, m d-d[) \text { for } m>1 .
$$

5.3.2. The strength of a singularity. We point out the following remark (see [14, Subsection 4.3): when studying the propagation and interaction of singularities in semilinear hyperbolic systems, Rauch and Reed in [36] defined the strength of a singularity of a piecewise smooth function. This notion is recalled in the one-dimensional case. Assume that the function $f: \mathbb{R} \rightarrow \mathbb{R}$ is smooth on $\left.]-\infty, x_{0}\right]$ and on $\left[x_{0}, \infty\left[\right.\right.$ for some point $x_{0} \in \mathbb{R}$. The strength of the singularity of $f$ at $x_{0}$ is the order of the highest derivative which is still continuous across $x_{0}$. For example, if $f$ is continuous with a jump in the first derivative at $x_{0}$, the order is 0 ; if $f$ has a jump at $x_{0}$, the order is -1 . Travers [39] later generalized this notion to include delta functions. Slightly deviating from his definition, but in line with the one of [36], it is possible to define the strength of singularity of the $k$-th derivative of the delta function at $x_{0}, \partial_{x}^{k} \delta\left(x-x_{0}\right)$, to be $-k-2$.

We can find the relation between the strength of a singularity of a function $f$ at $x_{0}$ and the singular spectrum of $\left[f * \varphi_{\varepsilon}\right]$, where $\varphi_{\varepsilon}$ is a convenient mollifier. More precisely we consider a function $f: \mathbb{R} \rightarrow \mathbb{R}$ which is smooth on $\left(-\infty, x_{0}\right]$ and on $\left[x_{0}, \infty\right)$ for some point $x_{0} \in \mathbb{R}$; actually only the local behaviour near $x_{0}$ is relevant. A mollifier $\varphi_{\varepsilon}(x)=\frac{1}{\varepsilon} \varphi\left(\frac{x}{\varepsilon}\right)$ is fixed as in 5.3.1 and the corresponding embedding of $\mathcal{D}^{\prime}(\mathbb{R})$ into the $(\mathcal{C}, \mathcal{E}, \mathcal{P})$-algebra $\mathcal{A}(\mathbb{R})$ is denoted by $\iota$. In particular, $\iota(f)=\left[f * \varphi_{\varepsilon}\right]$. If $f$ is continuous at $x_{0}$, then $\lim _{\varepsilon \rightarrow 0} f * \varphi_{\varepsilon}=f$ in $\mathrm{C}^{0}$. If $f$ has a jump $x_{0}$, this limit does not exist in $\mathrm{C}^{0}$, but $\lim _{\varepsilon \rightarrow 0} \varepsilon^{r} f * \varphi_{\varepsilon}=0$ in $\mathrm{C}^{0}$ for every $r>0$. Then we have the following result:

Proposition 5.7. Let $x_{0} \in \mathbb{R}$. If $f: \mathbb{R} \rightarrow \mathbb{R}$ is a smooth function on $\left(-\infty, x_{0}\right]$ and on $\left[x_{0}, \infty\right)$ or $f(x)=\partial_{x}^{k} \delta\left(x-x_{0}\right)$ for some $k \in \mathbb{N}$, then the strength of the singularity of $f$ at $x_{0}$ is $-n$ if and only if

$$
\Sigma_{\left(a, \mathrm{C}^{1}\right), x_{0}}(\iota(f))=[0, n] .
$$

Here $n \in \mathbb{N}$ and $a_{\varepsilon}(r)=\varepsilon^{r}$.

\section{References}

[1] J. Aragona and H. Biagioni, Intrinsic definition of the Colombeau algebra of generalized functions, Anal. Math. 17 (1991), 75-132.

[2] M. Beals, Propagation of smoothness for nonlinear second order strictly hyperbolic differential equations, A.M.S. Proc. Symp. Pure Math. 43 (1985), 21-44. 
[3] M. Beals, Propagation and interaction of singularities in nonlinear hyperbolic problems, in: Progress in Nonlinear Differential Equations and their Applications 3, Birkhäuser Boston, Boston, 1989.

[4] J.-M. Bony, Calcul symbolique et propagation des singularités pour les équations aux dérivées partielles non linéaires, Ann. Sc. Ec. Norm. Sup. 14 (1981), 209-246.

[5] J.-M. Bony, Analyse microlocale des équations aux dérivées partielles non linéaires, in: Lecture Notes in Math. 1495, Springer-Verlag, Berlin, 1991, 1-45.

[6] C. Bouzar and K. Benmeriem, Colombeau generalized Gevrey ultradistributions and their microlocal analysis, arXiv:0707.0124 vl [math. FA], 2007.

[7] J. F. Colombeau, Multiplication of Distributions: a tool in mathematics, numerical engineering and theoretical physics, Lecture Notes in Mathematics 1532, Springer-Verlag, Berlin, 1992.

[8] J. F. Colombeau, Generalized functions and infinitesimals, arXiv:0610264 [math. FA], 2006.

[9] J. F. Colombeau, Generalized functions as a tool for nonsmooth nonlinear problems in mathematics and physics, arXiv:0612077 [math.-ph.], 2006.

[10] J. F. Colombeau, Nonlinear generalized functions and nonlinear numerical simulations in fluid and solid continuum mechanics, arXiv:0702014 [math.-ph.], 2007.

[11] N. Dapić, S. Pilipović and D. Scarpalézos, Microlocal analysis of Colombeau's generalized functions: propagation of singularities, J. Anal. Math. 75 (1998), 51-66.

[12] A. Delcroix, Regular rapidly decreasing nonlinear generalized functions. Application to microlocal regularity, J. Math. Anal. Appl. 327 (2007), 564-584.

[13] A. Delcroix and J.-A. Marti, $\mathcal{G}^{r, \mathcal{R}, L}$-microlocal analysis of generalized functions, preprint.

[14] A. Delcroix, J.-A. Marti and M. Oberguggenberger, Microlocal asymptotic analysis in algebras of generalized functions, arXiv:0704.1077vl [math. FA], 2007.

[15] C. Garetto, T. Gramchev and M. Oberguggenberger, Pseudo-differential operators and regularity theory, Electron J. Diff. Eqns. 116 (2005), 1-43.

[16] C. Garetto, Topological structures in Colombeau algebras: topological $\widetilde{\mathbb{C}}$-modules and duality theory, Acta Appl. Math. 80 (2005), 81-125.

[17] C. Garetto, Microlocal analysis in the dual of a Colombeau algebra: generalized wave front sets and noncharacteristic regularity, New York J. Math. 12 (2006), 275-318.

[18] C. Garetto and G. Hörmann, Microlocal analysis of generalized functions: pseudodifferential techniques and propagation of singularities, Proc. Edinb. Math. Soc. 48 (2005), 603-629.

[19] M. Grosser, M. Kunzinger, M. Oberguggenberger and R. Steinbauer, Geometric Theory of Generalized Functions with Applications to General Relativity, Kluwer Academic Publ., Dordrecht, 2001.

[20] R. Godement, Topologie algébrique et théorie des faisceaux, Hermann, Paris, 1958.

[21] L. Hörmander, The Analysis of Linear Partial Differential Operators, I: Distribution Theory and Fourier Analysis, Grundlehren der mathematischen Wissenschaften 256, 2nd edition, Springer-Verlag, Berlin, 1990.

[22] L. Hörmander, Fourier integral operators I, Acta Math. 127 (1971), 79-183.

[23] L. Hörmander, Lectures on Nonlinear Hyperbolic Differential Equations, Springer-Verlag, Berlin, 1997.

[24] G. Hörmann and M. V. De Hoop, Microlocal analysis and global solutions for some hyperbolic equations with discontinuous coefficients, Acta Appl. Math. 67 (2001), 173-224.

[25] G. Hörmann and M. Kunzinger, Microlocal properties of basic operations in Colombeau algebras, J. Math. Anal. Appl. 261 (2001), 254-270. 
[26] G. Hörmann, M. Oberguggenberger and S. Pilipović, Microlocal hypoellipticity of linear differential operators with generalized functions as coefficients, Trans. Amer. Math. Soc. 358 (2006), 3363-3383.

[27] J.-A. Marti, $(\mathcal{C}, \mathcal{E}, \mathcal{P})$-Sheaf structure and applications, in: M. Grosser, G. Hörmann, M. Kunzinger, M. Oberguggenberger (eds.), Nonlinear Theory of Generalized Functions, Chapman \& Hall/CRC Research Notes in Mathematics 401, Boca Raton, 1999, 175-186.

[28] J.-A. Marti, Nonlinear algebraic analysis of delta shock wave to Burgers' equation, Pacific J. Math. 210 (2003), 165-187.

[29] J.-A. Marti, $\mathcal{G}^{L}$-microlocal analysis of generalized functions, Integral Transforms Spec. Funct. 2-3 (2006), 119-125.

[30] J.-A. Marti, Regularity, local and microlocal analysis in theories of generalized functions, arXiv:0711.368 [math. FA], 2007.

[31] M. Nedeljkov, S. Pilipović and D. Scarpalézos, The Linear Theory of Colombeau Generalized Functions, Pitman Research Notes in Mathematics 385, Longman Scientific \& Technical, Harlow, 1998.

[32] M. Oberguggenberger, Multiplication of Distributions and Applications to Partial Differential Equations, Pitman Research Notes in Mathematics 259, Longman Scientific \& Technical, Harlow, 1992.

[33] M. Oberguggenberger, Generalized solutions to nonlinear wave equations, Matemática Contemporânea 27 (2004), 169-187.

[34] S. Pilipović, D. Scarpalézos and V. Valmorin, Real analytic generalized functions, preprint.

[35] J. Rauch, Singularities of solutions to semilinear wave equation, J. Math. Pures et Appl. 58 (1979), 299-308.

[36] J. Rauch and M. Reed, Jump discontinuities of semilinear, strictly hyperbolic equations in two variables: Creation and propagation, Comm. Math. Phys. 81 (1981), 203-227.

[37] D. Scarpalézos, Colombeau's generalized functions: topological structures; microlocal properties. A simplified point of view, Bull. Cl. Sci. Math. Nat. Sci. Math. 25 (2000), 89-114.

[38] L. Schwartz, Théorie des Distributions, Hermann, Paris, 1966.

[39] K. Travers, Semilinear hyperbolic systems in one space dimension with strongly singular initial data, Electron. J. Diff. Eqns. 14 (1997), 1-11. 